\title{
Qualificação profissional e o câncer infantojuvenil na atenção básica
}

\author{
Professional qualification and childhood cancer in primary care \\ Cualificación profesional y cáncer infantojuvenil en la Atención Básica \\ Jane Kelly Oliveira Friestino $0^{1,2}$ (c) https://orcid.org/0000-0002-5432-9560

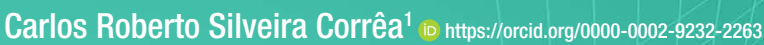 \\ Ana Izabel Jatobá de Souza ${ }^{3}$ ø nttps://orcid.org/0000-0003-3843-6144 \\ Graciela Soares Fonsêca ${ }^{1}$ ic https://orcid.org/0000-0001-9506-0409 \\ Ana Caroline Geremia ${ }^{1}$ ib https://orcid.org/0000-0002-0825-1344 \\ Djalma de Carvalho Moreira Filho² ID https://orcid.org/0000-0002-7943-0868
}

Como citar: Gouza Al, Fonsêca GS, Geremia AC, Moreira Filho DC. Qualificação profissional 0 o câncer infantojuvenil na atenção básica. Acta Paul Enferm. 2022;35:eAPE02771.

DOI

http://dx.doi.org/10.37689/acta-ape/2022A002771

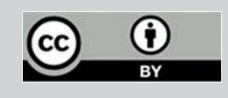

Descritores

Capacitação profissional; Atenção primária à saúde Neoplasias; Saúde da criança; Saúde do adolescente

Keywords Professional training; Primary health care; Neoplasms; Child health; Adolescent health

Descriptores

Capacitación professional; Atención primaria de salud Neoplasias; Salud del niño; Salud del adolescente

Submetido 30 de Setembro de 2020

Aceito

26 de Maio de 202

\section{Autor correspondente \\ Jane Kelly Oliveira Friestino} E-mail: jane.friestino@uffs.edu.br

Editor Associado (Avaliação pelos pares): Denise Myuki Kusahara (https://orcid.org/0000-0002-9498-0868) Escola Paulista de Enfermagem, Universidade Federal de São Paulo, São Paulo, SP, Brasil

\section{Resumo}

Objetivo: Analisar a percepção de profissionais que atuam na Atenção Básica sobre sua formação profissional relacionada ao câncer infantojuvenil.

Métodos: Trata-se de um estudo exploratório, com abordagem qualitativa, desenvolvido junto aos profissionais da Atenção Básica do município de Campinas-SP. A condução do estudo foi realizada por meio de grupos focais, orientados por questões semiestruturadas. Adotou-se a modalidade temática da Análise de Conteúdo.

Resultados: Foram identificadas duas categorias: "Experiências e formação profissional frente ao câncer infantojuvenil na Atenção Básica", traduzindo-se em pouco contato com a temática, tanto por experiências, quanto por meio da formação profissional; e, a "Integralidade do cuidado à criança e ao adolescente com câncer na Atenção Básica e a qualificação profissional", sendo desvelado pouco ou o nenhum preparo para garantir a integralidade do cuidado, com questões distintas dos aspectos biológicos da doença.

Conclusão: A partir das percepções dos profissionais, notou-se pouco contato e preparo insuficiente para elencar ações assertivas relacionadas ao câncer infantojuvenil na Atenção Básica, apontando necessidades de mudanças futuras na inclusão do tema neste nível de atenção e melhorias na qualidade da educação permanente nos serviços.

\section{Abstract}

Objective: To analyze the perception of professionals working in Primary Care about their professional training related to childhood cancer.

Methods: This is an exploratory study, with a qualitative approach, developed with professionals in Primary Care in the city of Campinas-SP. The study was conducted through focus groups, guided by semi-structured questions. Thematic modality of Content Analysis was adopted.

Results: Two categories were identified: "Experiences and professional training in the face of childhood cancer in Primary Care", resulting in little contact with the theme, both through experiences and through professional training; and "Comprehensiveness of care for children and adolescents with cancer in Primary Care and professional qualification", with little or no preparation being unveiled to ensure comprehensive care, with issues different from the biological aspects of the disease.

Conclusion: From professionals' perceptions, there was little contact and insufficient preparation to list assertive actions related to childhood cancer in Primary Care, pointing out the need for future changes in the inclusion of the theme in this level of care and improvements in the quality of continuing education in the services. 


\section{Resumen}

Objetivo: Analizar la percepción de profesionales que actúan en la Atención Básica sobre su formación profesional relacionada con el cáncer infantojuvenil.

Métodos: Se trata de un estudio exploratorio, con enfoque cualitativo, llevado a cabo con profesionales de la Atención Básica del municipio de Campinas, estado de São Paulo. El estudio fue conducido por medio de grupos focales, guiados con preguntas semiestructuradas. Se adoptó la modalidad temática del análisis de contenido.

Resultados: Se identificaron dos categorías: "Experiencias y formación profesional frente al cáncer infantojuvenil en la Atención Básica”, que se tradujo en poco contacto con la temática, tanto por experiencias, como mediante la formación profesional, e "Integralidad del cuidado de niños y adolescentes con cáncer en la Atención Básica y la cualificación profesional”, que reveló poco o ningún tipo de preparación para garantizar la integralidad del cuidado, con cuestiones distintas a los aspectos biológicos de la enfermedad.

Conclusión: A partir de las percepciones de los profesionales, se observó poco contacto y preparación insuficiente para enumerar acciones asertivas relacionadas con el cáncer infantojuvenil en la Atención Básica, lo que indica la necesidad de cambios futuros respecto a la inclusión del tema en este nivel de atención y mejoras en la calidad de la educación permanente en los servicios.

\section{Introdução}

A formação de profissionais, assim como a promoção de educação permanente para qualificação do cuidado, são as referências e metas dos movimentos que visam implementar estratégias que fortaleçam a promoção de cuidados efetivos nos diferentes níveis da atenção à saúde. ${ }^{(1,2)}$

Um dos caminhos possíveis para se realizar a organização dos serviços é a inclusão de propostas de trabalhos articulados, colocando em evidência a formação para a área da saúde, com base na junção entre desenvolvimento individual e institucional, o papel dos serviços e da gestáo setorial, e entre atenção à saúde e controle social. ${ }^{(3)}$

Com o propósito de garantir a integralidade do cuidado, busca-se uma formação interprofissional, alinhada às necessidades de saúde das pessoas e das populaçôes, sendo, capaz de impactar e influenciar na formulação de políticas de reorientação da formação em saúde. ${ }^{(3)}$

Do mesmo modo, é necessário que a estruturação, organização, e execução das ações e serviços do Sistema Único de Saúde brasileiro (SUS) sejam ordenadas pela Atenção Básica em todas as dimensóes, constituindo-se como porta principal de entrada do sistema e alicerce da atenção à saúde. ${ }^{(4)}$

Torna-se imprescindível que a Atenção Básica possua ações voltadas para ampliação do acesso aos outros níveis de complexidade do SUS. ${ }^{(5)}$ Como também apresenta-se necessário estabelecer uma formação adequada, produzindo-se conhecimento das peculiaridades e necessidades da população, e atendendo à complexidade dos processos saúde- -doença nos diferentes ciclos de vida, e sua interlocução com as questóes do território. ${ }^{(6)}$

Nessa vertente, no ano de 2013, foi instituída a Política Nacional para a Prevenção e Controle do Câncer na Rede de Atenção à Saúde das Pessoas com Doenças Crônicas ${ }^{(2)}$ que descreve a implementação de ações de diagnóstico precoce e de identificação dos sinais e sintomas de cânceres passíveis desta ação como uma das competências da Atenção Básica.

Com isso, a equipe de saúde que atua nesse nível de atenção torna-se responsável pela condução de açóes frente à prevenção e controle do câncer, como também, para garantir a integralidade do cuidado. Por conseguinte, muitas atividades específicas já têm sido desenvolvidas na Atenção Básica em relação a prevenção e controle do câncer, notoriamente quando se trata de saúde da mulher e saúde do homem, com um direcionamento expressivo à prevenção e controle de câncer de colo de útero e mama e câncer de próstata, apontados inclusive, pela agenda de açóes anuais, representadas pelos meses de outubro rosa e novembro azul. ${ }^{(7,8)}$

No entanto, quando se trata de açóes voltadas ao câncer na população infantojuvenil, existem poucas iniciativas para subsidiar a atuação dos profissionais da Atenção Básica, sendo consideradas temáticas e cuidados próprios das especialidades oncológicas, muitas vezes focalizadas somente em aspectos clínicos, desconsiderando a importância em incluir também aos profissionais que não executam o diagnóstico. ${ }^{(9)}$ Desse modo, pontua-se a relevância em se estabelecer modelos de atenção que reforcem a educação em saúde, objetivando ampliar a capaci- 
dade de intervenção de profissionais e também das pessoas em relação ao câncer infantojuvenil. ${ }^{(3,9)}$

Sabe-se que a ocorrência de câncer em crianças e adolescentes é considerada rara quando comparado aos adultos, ainda assim, é a primeira causa de morte por doenças em crianças de 0 a 19 anos. ${ }^{(10)}$ Os aspectos próprios do câncer infantojuvenil, ou seja, baixa incidência e sinais e sintomas inespecíficos, podem ser considerados condiçôes de difícil identificação para profissionais que atuam na Atenção Básica, cujo contato com eventos semelhantes não é frequente. ${ }^{(9,11)}$

Dentre as formas encontradas para minimizar os agravos decorrentes da doença e o impacto por ela provocado, encontram-se a suspeita e o diagnóstico precoce e, nesse sentido, a Atenção Básica possui um papel fundamental, tanto na realização da escuta qualificada, quanto no atendimento baseado em vínculo com toda a família, permitindo assim uma contínua articulação na Rede de Atenção à Saúde (RAS), efetuando encaminhamentos assertivos aos centros especializados, ampliando a possibilidade de início rápido do tratamento, o que, por sua vez, acarretam um melhor prognóstico e a redução de mortalidade por estas causas. ${ }^{(12,13)}$

Sendo assim, a presente investigação faz parte de um projeto maior, intitulado Crianças com câncer é difícil diagnosticar? Um estudo sobre a temática na Atenção Básica, e objetiva analisar a percepção de profissionais que atuam nessa esfera sobre sua formação profissional relacionada ao câncer infantojuvenil.

\section{Métodos}

Trata-se de um estudo exploratório, com abordagem qualitativa, norteado pela pesquisa social ${ }^{(14)}$ desenvolvido junto aos profissionais da Atenção Básica do município de Campinas, situado no estado de São Paulo, Brasil.

Campinas possui, aproximadamente, 1.182.429 de habitantes, ${ }^{(15)}$ atendidos por 63 Centros de Saúde (CS), distribuídos em 05 Distritos de Saúde (DS): Norte, Sul, Sudoeste, Noroeste e Leste. Estes CS, organizados a partir do modelo ampliado da
Estratégia Saúde da Família (ESF), atendendo em torno de 20.000 habitantes, cada um deles.

Vale ressaltar que a Atenção Básica do município, adota o modelo de apoio Paidéia, com territorialização, trabalho em equipe, apoio matricial, clínica ampliada e projeto terapêutico singular, ${ }^{(16)}$ contando com profissionais distintos, adaptados de acordo com as especificidades locais.

O cenário do estudo foi composto por $04 \mathrm{CS}$ que apresentaram população adscrita, instalações físicas, demanda de atendimento e recursos humanos semelhantes, com a característica de estarem distantes um dos outros. Desta maneira, cada CS selecionado esteve localizado em um DS diferente.

Antes da realizaçáo da coleta de dados, os pesquisadores realizaram visitas aos CS, com intuito de apresentar o estudo nas reunióes de equipe, permitindo que os profissionais conhecessem a pesquisa. Esse momento de aproximação inicial permitiu tomar nota a respeito das categorias profissionais dos participantes, bem como, do tempo de atuação destes na unidade.

Foram incluídos no estudo os trabalhadores que compóem a ESF, compreendendo categorias profissionais de nível médio e superior. Após o convite, participaram os profissionais que manifestaram interesse, sendo a amostra definida por conveniência. Foram excluídos aqueles que atuavam há menos de 06 meses na unidade, conforme figura 1 .

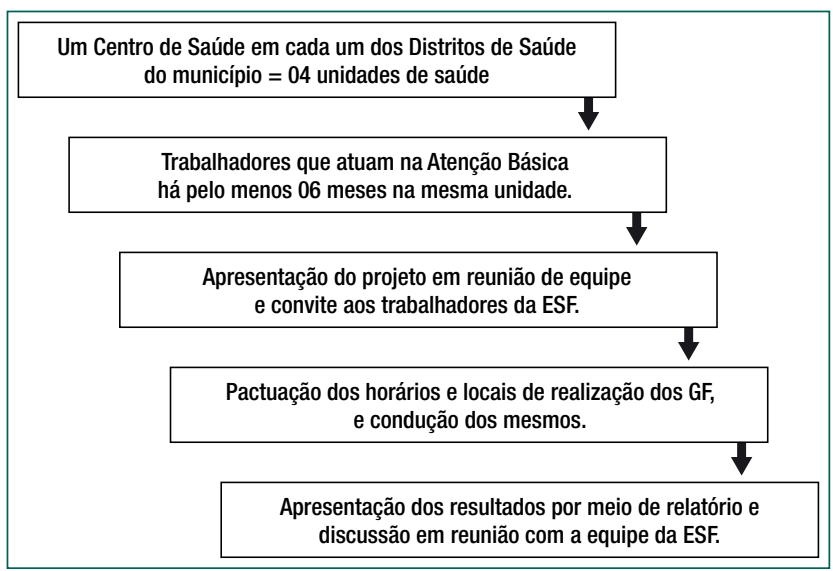

Figura 1. Fluxo de organização do trabalho de campo, recrutamento, realização e devolutiva do estudo

O estudo foi conduzido por meio de Grupos Focais $(\mathrm{GF}){ }^{(17)}$ realizados em salas dos próprios CS 
e em horários pactuados com a gestão das unidades de saúde, para que náo houvesse prejuízo no atendimento do serviço. Não foram vivenciadas interferências durante os GF.

Os registros foram feitos por meio de gravaçóes de áudio, e para identificar os participantes, no início de cada fala estes se apresentavam dizendo sua categoria profissional.

Todos os GF foram conduzidos a partir de um mesmo roteiro semiestruturado, contendo os seguintes temas: $\mathrm{O}$ câncer infantojuvenil na formação profissional e as experiências na Atenção Básica.

Cada GF teve duração média de 35 minutos, e antes do término de cada um deles, foi feita uma validação que congregou uma síntese das discussões realizadas. Todos os GF foram moderados por uma enfermeira mestre em saúde coletiva com experiência na condução de GF, favorecendo um enfoque pontual, sendo obtidas 02 horas e 19 minutos de conteúdo registrado, das quais foram produzidas 34 páginas de material transcrito manualmente. Não houve critério de saturação.

As etapas de transcrição, validação interna e análise, foram realizadas de forma independente por três pesquisadores envolvidos na pesquisa, incluindo pesquisadora que participou da coleta, um médico doutor em Saúde Coletiva e uma cirurgiã-dentista doutora em Odontologia social, todos com experiência na temática. Os pesquisadores não adotaram um software para apoiar a análise dos dados qualitativos. Ressalta-se que não houve perda nas gravaçóes ou problemas durante a coleta.

O material produzido foi baseado na transcrição na íntegra dos GF, sendo posteriormente analisados pela modalidade temática da Análise de Conteúdo de Bardin, ${ }^{(18)}$ de acordo com as seguinte percurso: reunião do corpus de análise, realização de leitura flutuante dos achados, leitura aprofundada, constituição e análise interpretativa das categorias e discussão com a literatura pertinente. A partir da análise emergiram duas categorias centrais: "Experiências e formação profissional frente ao câncer infantojuvenil na Atenção Básica" e a "Integralidade do cuidado à criança e ao adolescente com câncer na Atenção Básica e a qualificação profissional". Os participantes tiveram acesso aos resultados da pesquisa por meio do relatório final de pesquisa, discutidos nas reuniôes de equipe das unidades e disponibilizados à gestáo local e municipal.

Para a execução do estudo foram obedecidos os preceitos éticos da Resolução do Conselho Nacional de Saúde n ${ }^{\circ} 466 / 2012$, sendo o projeto de pesquisa aprovado pelo Comitê de Ética em Pesquisas da Universidade Federal de São Paulo - UNIFESP/ EPM (CAAE: 22920213.5.0000.5505).

\section{Resultados}

Foram realizados 04 GF com participação de 27 profissionais de nível médio e superior que atuam na Atenção Básica: 03 enfermeiras, 06 médicos, 01 cirurgiâo-dentista, 06 auxiliares de enfermagem, 01 educador popular e 10 Agentes Comunitárias de Saúde (ACS).

Após as etapas de transcrição das falas advindas dos GF, organização do corpus de análise, leitura flutuante seguida de leitura aprofundada, emergiram duas categorias de análise, conforme figura 2.

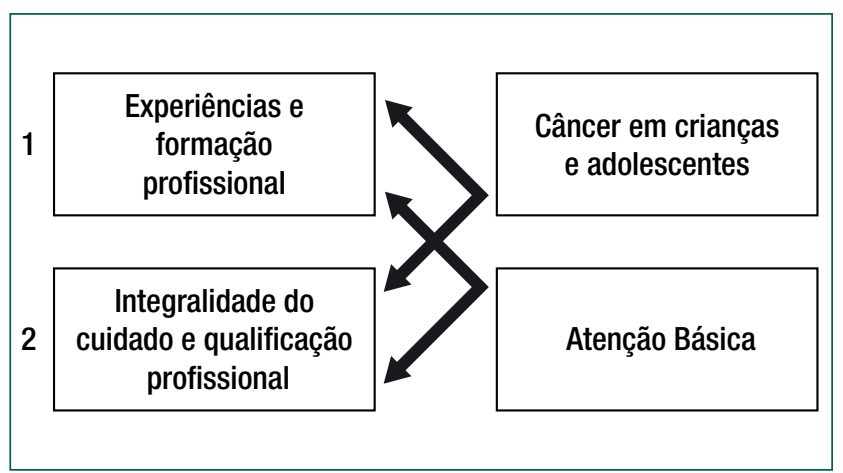

Figura 2. Temas emergentes dos Grupos Focais que contribuíram para formação das categorias de análise

\section{Experiências e formação profissional frente ao câncer infantojuvenil na Atenção Básica}

Foram investigados profissionais de nível médio e superior e, em suas falas, percebe-se que, em relação ao diagnóstico e tratamento/cuidado do câncer na infância e adolescência, houve pouco contato com a temática, tanto em relação às experiências, quanto por meio da formação profissional. Os recortes que seguem exemplificam essa inferência: 
Eu não tive absolutamente nada, no máximo uns slides na faculdade (Enfermeira) (GF 3).

Não, nunca tivemos nada (ACS) (GF 2).

Não tive nada (ACS) (GF 4).

Os profissionais da Atenção Básica relatam ter pouco conhecimento a respeito do câncer infantojuvenil, resumido em poucas aulas durante a graduação, e quando o tema foi apresentado se baseou em casos rápidos, não possibilitando um real aprendizado:

Tive uma abordagem muito pequena na oncologia geral. Mesmo eu tendo estudado em um lugar muito bom na área de oncologia, com crianças vi pouquissimos casos.. mesmo assim, foi bem limitado (Médica de Familia) (GF 4).

A minha formação de técnica de enfermagem foi bem superficial, pois nem mesmo nos estágios tive contato, na verdade, no técnico eu nem fui para a pediatria propriamente dita (Técnica de Enfermagem) (GF 4).

Eu também tive muito pouco know-how, na verdade, eu não tive! Nas nossas aulas na graduação, na pediatria, nós só tivemos um caso, e em um caso só, você não vê nada (Médica preceptora de clínica médica) (GF 4).

$\mathrm{Na}$ Atenção Básica, o profissional enfermeiro acompanha a longitudinalidade do cuidado individual e familiar, além de atuar na gestão dos serviços de saúde. Contudo, em suas percepçóes, sente-se despreparado para atender casos de câncer infantojuvenil, incluindo a implementação de açóes de diagnóstico precoce e de identificação dos sinais e sintomas.

A minha formação como enfermeira focou o cuidado, mas foi muito superficial em termos de câncer infantil, quase nada. Eu acho que o profissional de saúde, como um todo, ainda não está preparado. (Enfermeira) (GF 4).
Em relação aos ACS, percebe-se que as informaçôes da realidade não são problematizadas junto às organizaçôes de saúde, e a construção de significados para suas práticas é realizada sem o apoio institucional, congregando em um conhecimento que advém de suas experiências pessoais, e pouco contextualizadas com a organização das políticas e da própria Rede de Atenção à Saúde (RAS).

Na minha formação de ACS, a gente não viu nada, não tem nada sobre câncer em criança, nem sobre morte. Nada! O que a gente tem de experiência é na vivência como ser humano, de parentes e vizinhos. Mas dizer que aprendi isso... estudando isso... não! (ACS) (GF 4).

Em alguns casos, os profissionais frisaram que a aprendizagem relacionada à abordagem do câncer infantojuvenil ocorreu em função de experiências pessoais, conforme os recortes que seguem:

Quando eu ajudei essa criança que contei, eu sabia que precisava fazer aquilo pela minha experiência com minha filha, mas não foi dado nada aqui não, como por exemplo: como a gente deve abordar ou como a gente [deve] fazer... (ACS) (GF 2).

Eu nunca tive...aprendi com a vida (Auxiliar de Enfermagem) (GF4).

Ai você aprenda a diagnosticar, VOCÊ que se vire (Médica de Família) (GF 3).

Os profissionais verbalizaram, durante os GF, a importância de atividades de formação relacionada à abordagem do câncer infantojuvenil após a inserção no mundo do trabalho:

Mas, acho que sempre tem que ter reciclagem pois é uma necessidade que temos. Esse é um tema complicado, até o pediatra não gosta de fazer esse diagnóstico. No passado, tivemos umas reciclagens grandes, muito boas... acho que isso é importante, tem que reviver esse tema, não tem pediatra que tem facilidade com isso, eu sempre tive uma formação boa, foi discutido, mas não o suficiente (Pediatra) (GF 1). 
Na minha área (odontologia), é muito raro mesmo, a ênfase é em adultos e idosos. Todo ano a gente tem reciclagem, mas sempre relacionada ao adulto, por exemplo: tabagistas, alcoolistas (Cirurgiāodentista) (GF 1).

O que eu sei é o que aprendi aqui, na nossa educação permanente, inclusive eu vejo assim (Médica preceptora de clinica médica) (GF 4).

\section{A integralidade do cuidado à criança e ao adolescente com câncer na Atenção Básica e a formação profissional}

A garantia da integralidade do cuidado em saúde advém de um processo articulado de organização dos serviços e implica na necessidade de uma atenção que conjugue o trabalho colaborativo, em equipe, e em diferentes níveis de complexidade.

Os participantes do estudo apontaram dificuldades para abordagem do câncer infantojuvenil que se encontram para além do diagnóstico. Nos excertos registrados na sequência, é desvelado pouco ou o nenhum preparo para garantir a integralidade do cuidado, com questôes distintas dos aspectos biológicos da doença:

Como lidar com isso, de lidar com os pais, de lidar com a criança, de lidar com nós mesmos, e, lidar com a sua própria angústia? Então, quando pensamos em lidar com a criança com câncer isso fica a desejar.. (Médica de Família) (GF3).

Hoje está faltando muito essa questão de trabalhar o tato do sentimento do paciente, pois fica tudo em função do diagnóstico, dos exames, e no que isso vai desencadear. Mas como a gente vai trabalhar? Lidar com isso? E como a familia conviverá com isso? Essas questóes ficam um pouco fora da grade curricular (Médica de Familia) (GF 3).

A carga maior na formação da gente foca nos aspectos clinicos... as tecnologias de como que a pessoa lida com o problema que ela tem, ou, como que a família lida, isso na minha opinião, é relativamente novidade aqui no Brasil. De uma maneira geral é deficiente (Médico de Família) (GF 1).
Nas [aulas] práticas que a gente tinha era só [debatido] o diagnóstico e nada além disso. (Médica de Familia) (GF 4).

A dificuldade percebida pelos profissionais é expressa com clareza em relação a forma como lidam com situaçôes voltadas ao câncer infantojuvenil, bem como a falta de humanização nas práticas direcionadas à esse grupo, isso porque não houve o devido preparo, obrigando a realizaçáo de um tratamento inteiramente voltado para o exame e medicaçóes, náo se olhando a criança como um todo.

Não aprendi na faculdade a lidar com a finitude, com cuidados paliativos, com aceitaçâo da morte... ainda mais com a criança. A gente tem essa dificuldade de fazer isso até para idoso, e, com criança é meio que inaceitável. A gente não está preparado para a morte, mesmo aquele profissional da saúde que lida com isso o tempo todo. Acho que a gente tem dificuldade, pois nós, enquanto profissionais, sabemos a progressão natural da doença, e a gente sofre por antecedência. Agora a família, ela se apega na pontinha da esperança de que vai curar, que vai dar certo [...] o profissional de saúde tem que estar mais preparado para preparar a familia a lidar com essa questão da morte, e doença, resiliência (Enfermeira) (GF 4).

O que nós não temos é a formação de cidadão, porque com essa nossa sociedade capitalista, a questão da espiritualidade é pouco colocada em discussão. Não da religião em si, como religiáo católica e etc, mas sim a questão da espiritualidade. A gente pensar sobre a morte e a vida são coisas que com a condição paliativa está começando a entrar nos curriculos. Internacionalmente, tem disciplinas que tratam de espiritualidade, e acho que por conta da gente náo ter, não estamos preparados para pensar estas coisas (Médica preceptora de clínica médica) (GF 4).

Na formação não se está muito preocupado com essa relação médico paciente, na relação humana (Médico de Família) (GF 3). 


\section{Discussão}

As falas revelaram lacunas existentes na formação dos profissionais da Atenção Básica, sendo que, de um modo geral, as percepçóes voltam-se ao pouco ou nenhum contato com a temática do câncer infantojuvenil. O processo de formação profissional deve favorecer o trabalho colaborativo em equipe, estabelecendo estratégias de aprendizagem que favoreçam o diálogo, a troca, a transdisciplinaridade entre os distintos saberes formais e não-formais que contribuam para as açóes de promoção de saúde a nível individual e coletivo. ${ }^{(19-21)}$

Os achados deste estudo corroboram com alguns exemplos já mencionados pela literatura, ${ }^{(9,11,22)}$ observando-se uma lacuna considerável relacionada à capacitação dos profissionais da Atenção Básica, com intensa carência sobre o tema, incluindo a enfermagem, que declaram ser necessárias estratégias de qualificação para que possam consequentemente identificar precocemente o câncer infantojuvenil. ${ }^{(11)}$ Para exemplificar, cita-se o trabalho de Rosa, ${ }^{(23)}$ que revelou um déficit de qualificação relacionado ao tema oncologia na Atenção Básica.

Ressalta-se que a experiência apresenta grande potencial de formação e transformação. ${ }^{(20)}$ É fundamental reconhecer que existe uma relação de complementaridade e de recomposiçáo entre o que se aprende na formação institucionalizada e a aprendizagem derivada da experiência. ${ }^{(22)}$ No entanto, deve-se viabilizar o contato inicial com temas de grande relevância, como o câncer infantojuvenil, na educaçáo formal. ${ }^{(23)}$

Aliada a essa concepção, vem o conceito de Educação Permanente em Saúde que visa promover aprendizagem a partir de problemáticas enfrentadas no cotidiano de trabalho, no sentido de encontrar respostas e transformar as práticas. ${ }^{(1,24)}$ Deseja-se que os profissionais aprimorem o cuidado oncológico, especialmente voltado para pacientes infantojuvenis, a partir do desenvolvimento de conhecimentos técnicos em esfera biológica, psicológica e emocional. ${ }^{(25)}$

A necessidade de um processo permanente de formação pode ser traduzida por uma visão de maior consciência ao incorporar novas responsabilidades, relacionando-se diretamente com o processo de tomada de decisão na prática profissional. ${ }^{(24)}$
Tais aspectos são fundamentais para que os profissionais estejam engajados e comprometidos com a saúde da população, daí a importante tarefa de identificar as lacunas de conhecimento e a formação voltada para a atenção oncológica. Diferente do adulto, o câncer infantojuvenil apresenta uma multiplicidade de sinais e sintomas que se assemelham a inúmeros eventos próprios da infância, ou seja, os primeiros sintomas do câncer infantil são considerados inespecíficos. ${ }^{(12)}$ Esse fato, associado à baixa prevalência dessa afecção em relação às outras doenças com as quais partilha sinais e sintomas, faz com que o valor preditivo positivo desses sintomas para o câncer infantojuvenil seja baixo, e consequentemente dificulta o diagnóstico precoce.

Quando se pensa na integralidade do cuidado à criança e ao adolescente com câncer na Atenção Básica, o trabalho colaborativo em equipe torna-se algo essencial, com destaque à formação centrada na atenção à saúde, que requer e a construção coletiva de saberes. ${ }^{(19)}$ A Educação Interprofissional (EIP) desponta como horizonte desejável no sentido de propiciar o desenvolvimento de competências comuns e colaborativas que qualifiquem o trabalho em saúde, em especial no âmbito das doenças crônicas. ${ }^{(21,26)}$

No entanto, com as influências do relatório Flexner e seus desdobramentos para os cursos na área da saúde, o conhecimento oferecido aos estudantes, predominantemente, baseia-se na formação uniprofissional e na compreensão do adoecimento restrito ao corpo biológico, disfuncionalizando-o em relaçáo à normalidade. ${ }^{(27)}$ Um corpo fragmentado em órgãos, compreendido por disciplinas isoladas e visto a partir de lesôes orgânicas, somente. ${ }^{(28)}$

Além disso, o imaginário técnico-científico da saúde incentiva o entendimento da história natural das doenças, não valorizando as singularidades do viver e do sentir. ${ }^{(29)}$ Essa constituição histórica explica porque boa parte dos profissionais de saúde apresentam uma formação insuficiente para desenvolver um trabalho pautado nos princípios do SUS. ${ }^{(30-32)}$

As deficiências na formação para a abordagem do câncer infantojuvenil, mesmo sendo esse um agravo marcado por ser a primeira causa de óbito por doenças nessa faixa etária, ${ }^{(1)}$ reforçam o quão distante a formação encontra-se das necessidades 
sociais. Levando em consideração que o diagnóstico precoce é fundamental para minimizar os impactos causados pela doença, ${ }^{(10)}$ é essencial que os profissionais de saúde construam conhecimentos, habilidades e competências voltadas para essa problemática durante a formação.

Assim, a abordagem coerente com essa compreensão é voltada à esfera técnica e biológica, com uso excessivo de tecnologias duras, em detrimento das tecnologias de cuidado leve-relacionais. ${ }^{(33)}$ Nota-se que a formação em saúde pouco tem se voltado para o ensino de práticas cuidadoras uma vez que não prioriza a compreensão do corpo vivo, subjetivado e singular que demanda mais que diagnóstico e técnica. ${ }^{(33)} \mathrm{A}$ convivência com o paciente requer escuta qualificada e uma atenção sensível e delicada, vinculadas ao seu contexto histórico, social e familiar, ${ }^{(34)}$ especialmente quando se trata de câncer infantojuvenil.

A atual Política Nacional de Educação Permanente em Saúde ${ }^{(1)}$ enfatiza a necessidade de que a qualificação dos trabalhadores tenha aderência às necessidades e realidades locais com o objetivo de contribuir para a transformação das práticas e organização do trabalho. A partir das categorias analisadas, reforça-se a relevância da Educação Permanente para qualificar as práticas de cuidado na Atenção Básica, apontando para a necessidade em estabelecer também uma agenda com foco específico aos sinais e sintomas do câncer nesse ciclo de vida.

Neste âmbito, há uma valorização de práticas pedagógicas centradas na problematização e nos processos de trabalho de saúde, com uma ênfase no trabalho colaborativo em equipe sem menosprezar a importância específica que cada profissão possui. ${ }^{\text {(35) }}$

Ao considerar as especificidades do câncer infantojuvenil, as lacunas de conhecimento desses profissionais podem ser ainda maiores, como evidenciado pelos discursos dos participantes. O compromisso de cada profissional deve ser visto de maneira ativa da práxis-ação e reflexão sobre a realidade, exigindo-se um aperfeiçoamento, de superação do especialismo, incluindo a ampliação dos seus conhecimentos em torno do homem, e de sua forma de ser no mundo. ${ }^{(29)}$

Uma limitação presente no estudo foi o modo de realização dos GF, tanto por terem sido conduzidos somente por uma pesquisadora, como também, pelo tempo reduzido de realização de cada um deles. Esse fato ocorreu, pois os GF somente puderam ser realizados nos horários em que o serviço de saúde não fosse prejudicado, o que inviabilizou a participaçáo de todos os investigadores. No entanto, essa limitação foi amenizada com a participação efetiva de três pesquisadores nas etapas de transcrição, análise e interpretação dos resultados.

\section{Conclusão}

A partir das percepçóes dos profissionais, notou-se pouco contato e preparo insuficiente para elencar ações assertivas relacionadas ao câncer infantojuvenil na Atenção Básica. Além disso, a qualificação dos profissionais apresentou-se muitas vezes ser baseada em experiências pessoais, com a presença de dificuldades em lidar em situaçóes voltadas ao câncer infantojuvenil, com pouco aprofundamento da análise dos problemas identificados na prática dos serviços. Os achados do estudo apontam para necessidades de: mudanças futuras na inclusão do tema do câncer infantojuvenil na Atenção Básica; melhorias na qualidade da educação permanente nos serviços; e, implementação de ações de diagnóstico precoce, a fim de promover a saúde integral de crianças, adolescentes, e suas famílias.

\section{Agradecimentos}

À Coordenação de Aperfeiçoamento de Pessoal de Nível Superior. Bolsa de doutorado e à Universidade Federal da Fronteira Sul Edital de fomento à PósGraduação 1010/GR/UFFS/2019.

\section{Colaborações}

Friestino JKO, Corrêa CRS, Souza AIJ, Fonsêca GS, Geremia AC, Moreira Filho DC colaboraram com a contribuição do estudo, análise e interpretação dos dados, redação do artigo, revisão crítica relevante do conteúdo intelectual e aprovação da versão final a ser publicada. 


\section{Referências}

1. Brasil. Ministério da Saúde. Portaria GM/MS № 1.996 de 20 de agosto de 2007. Dispõe sobre as diretrizes para a implementação da Política Nacional de Educação Permanente em Saúde. Brasília (DF): Ministério da Saúde; 2007 [citado 2020 Dez 20]. Disponível em: http://bvsms. saude.gov.br/bvs/saudelegis/gm/2007/prt1996_20_08_2007.html

2. Brasil. Ministério da Saúde. Portaria nº 874, de 16 de maio de 2013. Dispõe sobre a Política Nacional para a Prevenção e Controle do Câncer na Rede de Atenção à Saúde das Pessoas com Doenças Crônicas no âmbito do Sistema Único de Saúde. Brasília (DF): Ministério da Saúde; 2013 [citado 2020 Dez 30]. Disponível em: http://bvsms.saude.gov.br/ bvs/saudelegis/gm/2013/prt0874_16_05_2013.html

3. Freire Filho JR, Silva CB, Costa MV, Forster AC. Educação Interprofissional nas políticas de reorientação da formação profissional em saúde no Brasil. Saúde Debate. 2019;43(Spe 1):86-96.

4. Santos L. Healthcare regions and their care networks: an organizationalsystemic model for SUS. Cien Saude Colet. 2017;22(4):1281-9.

5. Bousquat A, Giovanella L, Campos EM, Almeida PF, Martins CL, Mota PH, et al. Primary health care and the coordination of care in health regions: managers' and users' perspective. Cien Saude Colet. 2017;22(4):1141-54.

6. Sant'Anna CF, Cezar-Vaz MR, Cardoso LS, Bonow CA, Silva MR. Community: collective objective of nurses' work within the Family Health Strategy. Acta Paul Enferm. 2011;24(3):341-7.

7. Gutiérrez MG, Almeida AM. Pink October [Editorial]. Acta Paul Enferm. 2017;30(5):3-5.

8. Modesto AA, Lima RL, D'Angelis AC, Augusto DK. Um novembro não tão azul: debatendo rastreamento de câncer de próstata e saúde do homem. Interface. 2018;22(64):251-62.

9. Lima IM. Câncer infantojuvenil: ações de enfermagem na atenção primária à saúde. Rev APS. 2018;21(2):197-205.

10. Brasil. Ministério da Saúde. Instituto Nacional de Câncer José Alencar Gomes da Silva (INCA). Incidência, mortalidade e morbidade hospitalar por câncer em crianças, adolescentes e adultos jovens no Brasil: informações dos registros de câncer do sistema de mortalidade. Rio de Janeiro: INCA; 2016 [citado 2020 Dez 30]. Disponível em: https://www.inca.gov.br/publicacoes/livros/incidencia-mortalidade-emorbidade-hospitalar-por-cancer-em-criancas-adolescentes

11. Miranda LR, Melaragno AL, Pina-Oliveira AA. Diagnóstico precoce do câncer infanto-juvenil na atenção primária à saúde e contribuições do enfermeiro: revisão da literatura. Rev Saúde. 2017;11(3-4):63-74. Review.

12. Handayani K, Sitaresmi MN, Supriyadi E, Widjajanto PH, Susilawati D, Njuguna $F$, et al. Delays in diagnosis and treatment of childhood cancer in Indonesia. Pediatr Blood Cancer. 2016;63(12):2189-96.

13. Brasil. Ministério da Saúde. Protocolo de diagnóstico precoce do câncer pediátrico. Brasília (DF): Ministério da Saúde; 2017 [citado 2021 Jan 20]. Disponível em: http://bvsms.saude.gov.br/bvs/publicacoes/ protocolo_diagnostico_precoce_cancer_pediatrico.pdf

14. Minayo MC, Deslandes SF, Gomes R. Pesquisa social: teoria, método e criatividade. Petrópolis: Vozes; 2009.

15. Instituto Brasileiro de Geografia e Estatística. Infográficos Cidades@ São Paulo, Campinas. Rio de Janeiro: IBGE; 2018 [citado 2021 Jan 20]. Disponível em: http://www.ibge.gov.br/cidadesat/painel/painel. php?codmun $=350950$

16. Oliveira MM, Campos GW. Formação para o Apoio Matricial: percepção dos profissionais sobre processos de formação. Physis. 2017;27(2):187-206
17. Souza MK, LimaYO, PazBM, CostaEA, CunhaAB, Santos R. Potencialidades da técnica de grupo focal para a pesquisa em vigilância sanitária e atenção primária à saúde. Rev Pesq Qualitat. 2019;7(13):57-71 .

18. Bardin L. Análise de conteúdo. São Paulo: Almedina; 2011. 280 p.

19. Casanova IA, Batista NA, Moreno LR. A educação interprofissional e a prática compartilhada em programas de residência multiprofissional em saúde. Interface. 2018;22(Suppl 1):1325-37.

20. Carvalho MS, Merhy EE, Sousa MF. Repensando as políticas de Saúde no Brasil: Educação Permanente em Saúde centrada no encontro e no saber da experiência. Interface. 2019;23:e190211.

21. Nuin JJ, Méndez MJ. Porque precisamos da educação interprofissional. In: Nuin JJ, Francisco El. Manual de educação interprofissional em saúde. Rio de Janeiro: Elsevier, 2019. p.13.

22. Barros S, Claro HG. The teaching-learning process in mental health: the student's perspective about psychosocial rehabilitation and citizenship. Rev Esc Enferm USP. 2011;45(3):700-7.

23. Rosa LM, Andrade AE, Berndt LK, Anders JC, Radünz V, Souza Al. Atenção Oncológica na Atenção Básica: projeto de extensão na formação de acadêmicos de enfermagem. Rev Eletr Extensão. 2017;14(26):107-18.

24. Cavalcanti FO, Guizardi FL. Educação continuada ou permanente em saúde? análise da produção Pan-americana da saúde. Trab Educ Saúde. 2018;16(1):99-122.

25. Rezende VM, Neves GF. Formação, treinamento e aperfeiçoamento em oncologia infantojuvenil no âmbito do Programa Nacional de Apoio à Atenção oncológica: um panorama do triênio 2016-2018. Rev Bras Cancerol. 2018;64(3):327-3.

26. Agreli HF, Peduzzi M, Silva MC. Patient centred care in interprofessional collaborative practice. Interface. 2016;20(59):905-16.

27. Flexner A. Medical education in the United States and Canada. From the Carnegie Foundation for the Advancement of Teaching, Bulletin Number Four, 1910. Bull World Health Organ. 2002;80(7):594-602.

28. Gonze GG, Silva GA. A integralidade na formação dos profissionais de saúde: tecendo valores. Physis. 2011;21(1):129-146.

29. Ceccim RB, Carvalho YM. Ensino da saúde na integralidade: a educação dos profissionais de saúde no SUS. In: Pinheiro R, Ceccim RB, Mattos RA, organizadores. Ensinar saúde: a integralidade e o SUS nos cursos de graduação na área da saúde. Rio de Janeiro: CEPESC, IMS/UFRJ, ABRASC0; 2011. p. 69-92.

30. Barbosa LG, Damasceno RF, Silveira DM, Costa SM, Leite MT. Recursos humanos e estratégia saúde da família no norte de minas gerais: avanços e desafios. Cad Saúde Colet. 2019;27(3):287-94.

31. Cunha MS, Alvarenga EC. Educação permanente para avaliação em saúde: uma proposta na arte do encontro. Saúde Redes. 2018;4(1):75-84.

32. Carnut L. Cuidado, integralidade e atenção primária: articulação essencial para refletir sobre o setor saúde no Brasil. Saúde Debate. 2017:41(115):1177-86.

33. Lima AA, Jesus DS, Silva TL. Densidade tecnológica e o cuidado humanizado em enfermagem: a realidade de dois serviços de saúde. Physis. 2018;28(3):e280320.

34. Fonsêca GS, Souza JV. Narrative of an educational path: providing a (new) meaning to medical education. Interface. 2019;23(Suppl 1):e180059.

35. Cardoso ML, Costa PP, Costa DM, Xavier C, Souza RM. The National Permanent Health Education Policy in Public Health Schools: reflections from practice. Cien Saude Colet. 2017;22(5):1489-500. 